\title{
Effects of MRI Protocol Parameters, Preload Injection Dose, Fractionation Strategies, and Leakage Correction Algorithms on the Fidelity of Dynamic-Susceptibility Contrast MRI Estimates of Relative Cerebral Blood Volume in Gliomas
}

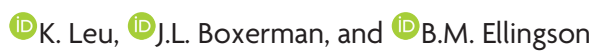

\begin{abstract}
BACKGROUND AND PURPOSE: DSC perfusion MR imaging assumes that the contrast agent remains intravascular; thus, disruptions in the blood-brain barrier common in brain tumors can lead to errors in the estimation of relative CBV. Acquisition strategies, including the choice of flip angle, TE, TR, and preload dose and incubation time, along with post hoc leakage-correction algorithms, have been proposed as means for combating these leakage effects. In the current study, we used DSC-MR imaging simulations to examine the influence of these various acquisition parameters and leakage-correction strategies on the faithful estimation of CBV.
\end{abstract}

MATERIALS AND METHODS: DSC-MR imaging simulations were performed in 250 tumors with perfusion characteristics randomly generated from the distributions of real tumor population data, and comparison of leakage-corrected CBV was performed with a theoretic curve with no permeability. Optimal strategies were determined by protocol with the lowest mean error.

RESULTS: The following acquisition strategies (flip angle/TE/TR and contrast dose allocation for preload and bolus) produced high CBV fidelity, as measured by the percentage difference from a hypothetic tumor with no leakage: 1) $35^{\circ} / 35 \mathrm{~ms} / 1.5$ seconds with no preload and full dose for DSC-MR imaging, 2) $35^{\circ} / 25 \mathrm{~ms} / 1.5$ seconds with $1 / 4$ dose preload and $3 / 4$ dose bolus, 3) $60^{\circ} / 35 \mathrm{~ms} / 2.0$ seconds with $1 / 2$ dose preload and $1 / 2$ dose bolus, and 4) $60 \% 35 \mathrm{~ms} / 1.0$ second with 1 dose preload and 1 dose bolus.

CONCLUSIONS: Results suggest that a variety of strategies can yield similarly high fidelity in CBV estimation, namely those that balance T1- and T2*-relaxation effects due to contrast agent extravasation.

ABBREVIATIONS: AIF = arterial input function; Bidir = bidirectional leakage correction algorithm; $C N R=$ contrast-to-noise ratio; EES = extravascular extracellular space; $K^{\text {trans }}=$ efflux rate of contrast agent from the vasculature; $r C B V=$ relative $C B V ;$ Unidir = unidirectional leakage correction algorithm

D SC-MR imaging is a PWI technique based on the indicatordilution theory, ${ }^{1}$ which uses the first pass of a paramagnetic contrast agent to estimate cerebrovascular parameters, including relative $\mathrm{CBV}$ ( $\mathrm{rCBV}$ ) and $\mathrm{CBF}^{2,3}$ A primary clinical application for rCBV includes the evaluation of brain tumor vascularity and

Received July 17, 2016; accepted after revision October 4.

From the University of California, Los Angeles Brain Tumor Imaging Laboratory (K.A.B.L., B.M.E.), Center for Computer Vision and Imaging Biomarkers; Department of Bioengineering (K.A.B.L., B.M.E.), Henry Samueli School of Engineering and Applied Science; and University of California, Los Angeles Neuro-Oncology Program (B.M.E.), University of California, Los Angeles, Los Angeles, California; Departments of Radiological Sciences (A.B.L., B.M.E.) and Biomedical Physics (B.M.E.), David Geffen School of Medicine, University of California, Los Angeles, Los Angeles, California; and Department of Diagnostic Imaging (J.L.B.), Rhode Island Hospital and Alpert Medical School of Brown University, Providence, Rhode Island.

This work was supported by the American Cancer Society Research Scholar Grant RSG-15-003-01-CCE (B.M.E.); the National Brain Tumor Society Research Grant

(B.M.E.); the Siemens Healthcare Research Grant (B.M.E.); and the University of California, Los Angeles Medical Scientist Training Program (K.A.B.L.).

Please address correspondence to Benjamin M. Ellingson, PhD, UCLA Brain Tumor Imaging Laboratory, Departments of Radiology, Biomedical Physics, Bioengineering, and Psychiatry, and Radiological Sciences and Psychiatry, David Geffen School of Medicine, University of California, Los Angeles, 924 Westwood Blvd, Suite 615, Los Angeles, CA 90024; e-mail: ellingson@mednet.ucla.edu angiogenesis; however, neovascularity within neoplasms tends to have elevated vascular permeability, resulting in contrast agent leakage into the extravascular extracellular space (EES) and violation of assumptions made by the indicator-dilution theory. These "leakage effects," which can be either T1weighted, which would cause underestimation of the rCBV, or $\mathrm{T} 2{ }^{\star}$-weighted, which would cause overestimation of the rCBV, greatly depend on the acquisition strategy and protocol used for DSC-MR imaging signal acquisition. ${ }^{4}$ To address these problems, strategies have been proposed for reducing the influence of contrast agent leakage, many focusing on T1weighted artifact reduction, including use of low flip angles, ${ }^{5}$ dual-echo acquisitions, ${ }^{6-8}$ preload administration, ${ }^{9}$ and/or postprocessing leakage-correction algorithms. ${ }^{10-13}$

Previous studies have used a combination of these strategies to reduce extravasation-induced error of CBV estimates; however,

Indicates article with supplemental on-line table.

Indicates article with supplemental on-line photo.

http://dx.doi.org/10.3174/ajnr.A5027 
these approaches have primarily been evaluated empirically. The goal of this study was to systematically evaluate, with simulation, the effects of various leakage-correction strategies on the fidelity of CBV estimation using simulated DSC-MR imaging data derived from the convolution theory ${ }^{14}$ and recent developments by Quarles et al. ${ }^{15}$ We hypothesized that this approach could provide insight into the interaction of pulse sequence parameters, preload dosing, and leakage-correction algorithms that are not readily determined experimentally.

\section{MATERIALS AND METHODS}

\section{Simulation Procedure}

The following common DSC-MR imaging protocol variables were evaluated by using simulations: pulse sequence parameters, including flip angle, TE, and TR; preload dose and incubation time; truncation of the DSC-MR imaging dataset after first pass to limit postbolus leakage contamination; and postprocessing leakage-correction algorithms. Simulated DSC-MR imaging signal curves for brain tumors were generated via the following: 1) selection of pulse sequence parameters; 2) construction of the DSC-MR imaging relaxivity-time-series without leakage for "ground truth rCBV"; 3) construction of the leakage-affected intravascular and EES contrast agent concentration-time-series based on tumor characteristics; and 4) estimation of CBV by using no leakage correction, unidirectional leakage correction (Unidir) as described by Boxerman et al, ${ }^{10}$ or bidirectional leakage correction (Bidir) accounting for bidirectional contrast agent flux between the vasculature and EES. ${ }^{11,13}$

\section{Simulated DSC-MR Imaging Relaxation Rate-Time Curve}

The simulated DSC-MR imaging relaxation rate-time curve is derived from the gradient-echo signal equation, which has signal contributions from both $\mathrm{T} 1$ and $\mathrm{T} 2$. When the MR imaging signal intensities, which have arbitrary units, are converted to $\left(\Delta R_{2}^{*}(\mathrm{t})\right.$, in units of $1 / \mathrm{s}$, these contributions are modeled for a single-shot gradient-echo EPI acquisition as the following ${ }^{15}$ :

1) $\Delta R_{2}^{*}(t)=r_{2, \mathrm{P}}^{*} v_{\mathrm{P}} C_{\mathrm{p}}(t)+r_{2, \mathrm{E}}^{*} v_{\mathrm{E}} C_{\mathrm{E}}(t)+K_{\mathrm{P}} v_{\mathrm{P}} v_{\mathrm{E}}\left|C_{\mathrm{P}}-C_{\mathrm{E}}\right|$

$$
\begin{aligned}
-\frac{1}{T E} \times( & \ln \frac{1-E_{1} \times e^{-T R \times r_{1} \times C_{\mathrm{T}}(t)}}{1-E_{1}} \\
& \left.-\ln \frac{1-\cos \alpha \times E_{1} \times e^{-T R \times r_{1} \times C_{\mathrm{T}}(t)}}{1-\cos \alpha \times E_{1}}\right)
\end{aligned}
$$

where $\alpha$ is the flip angle; $E_{1}=e^{-T R / T_{10}}$; subscripts E, I, and $\mathrm{P}$ represent the extravascular, intracellular, and plasma compartments, respectively; $v$ represents the volume fraction; $K$ represents "calibration susceptibility factors"; $C$ represents contrast agent concentration; and $r_{1}$ and $r_{2}^{*}$ are the T1 and $\mathrm{T} 2$ gadolinium relaxivities, respectively. The first term, $r_{2, \mathrm{P}}^{*} C_{\mathrm{p}}(t)$ represents the $\mathrm{T} 2{ }^{*}$ contribution of the plasma concentration of contrast agent; the second term, $r_{2, \mathrm{E}}^{\star} C_{\mathrm{E}}(t)$ represents the extravascular extracellular contrast agent contribution to $\mathrm{T}^{*} ; K_{\mathrm{P}} V_{\mathrm{P}} V_{\mathrm{E}}\left|C_{\mathrm{P}}-C_{\mathrm{E}}\right|$, represents the $\mathrm{T} 2{ }^{\star}$-weighted contribution owing to the difference in the concentration of contrast agent between the vasculature and the EES; and

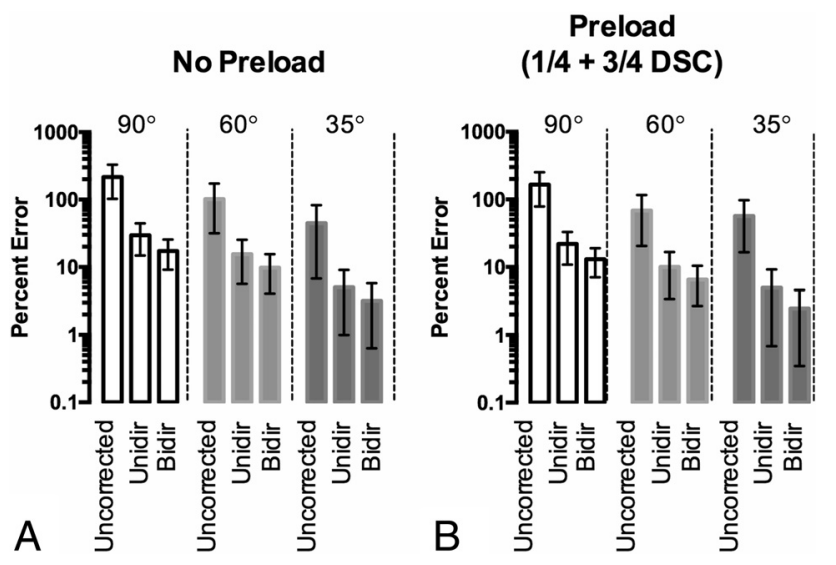

FIG 1. Effect of the flip angle on recovery of CBV $(T E=35 \mathrm{~ms} / \mathrm{TR}=$ 1.0 second). A, Percentage error (with $95 \% \mathrm{Cl}$ ) of the estimated CBV for different flip angles and leakage-correction strategies, without the use of preload, compared with ground truth CBV. B, Percentage error (with $95 \% \mathrm{Cl}$ ) of the estimated CBV for different flip angles and leakage-correction strategies, with use of $1 / 4$ dose preload, compared with ground truth CBV.

$-\frac{1}{T E} \times\left(\ln \frac{1-E_{1} \times e^{-T R \times r_{1} \times C_{\mathrm{T}}(t)}}{1-E_{1}}-\ln \frac{1-\cos \alpha \times E_{1} \times e^{-T R \times r_{1} \times C_{\mathrm{T}}(t)}}{1-\cos \alpha \times E_{1}}\right)$ represents the $\mathrm{T} 1$ contribution of contrast agent in both the plasma and EES.

\section{Pulse Sequence Parameters}

We tested all combinations of the following DSC-MR imaging parameters: flip angle $=35^{\circ}, 60^{\circ}$, and $90^{\circ} ; \mathrm{TE}=15,25,35,45$, and $55 \mathrm{~ms}$; TR $=1.0,1.5$, and 2.0 seconds; fractional preload + bolus dosage $=1 / 4+3 / 4(6 \mathrm{mmol} / \mathrm{L}$ total, single dose $), 1 / 2+1 / 2(6 \mathrm{mmol} / \mathrm{L}$ total, single dose), and $1+1$ (12 mmol/L total, double dose), in which a different value of peak arterial input function (AIF) concentration would simply scale all relaxivity-time curves proportionally.

\section{Construction of Blood Plasma and EES Concentration}

A generic AIF, which models the input of contrast agent into the tissue vasculature, was generated by using the following $\gamma$-variatelike approximation:

$$
C_{\alpha}(t)=A\left(t / t_{\mathrm{p}}^{2}\right) e^{-t / t_{\mathrm{p}}}+B\left(-e^{-t / t_{\mathrm{p}}}\right),
$$

where $A=200 \mathrm{mmol} / \mathrm{L} \mathrm{s}, B=1.75 \mathrm{mmol} / \mathrm{L}$, and $t_{\mathrm{p}}=2$ seconds (from Simpson et $\mathrm{al}^{16}$ ) and the peak concentration was 6.0 $\mathrm{mmol} / \mathrm{L}$ for the full dose and scaled appropriately for the preload dosages and postpreload bolus injections. For preload simulations, the composite AIF was constructed as the superposition of the preload injection AIF and the bolus AIF was delayed by the specified incubation time.

The blood plasma contrast agent concentration was computed by convolving the AIF with an exponential residue function, where the residue function describes the tracer retention; the convolution is used to describe the AIF as a series of narrow, instantaneous boluses of contrast agent; and the CBF factor accounts for the proportionality of the concentration in the vasculature to the delivered blood ${ }^{14}$ :

AJNR Am J Neuroradiol 38:478-84 Mar 2017 www.ajnr.org 
No Preload
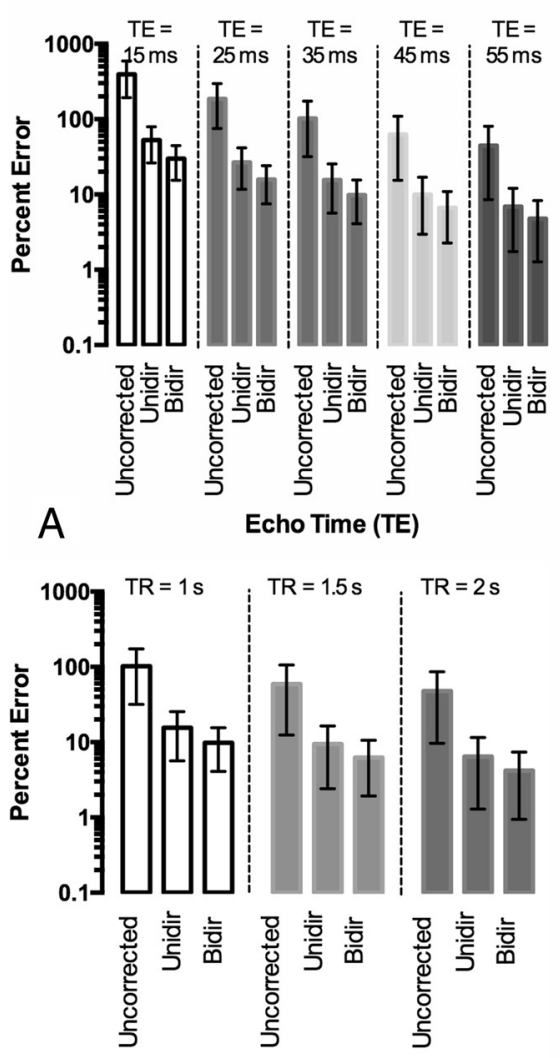

C

Repetition Time (TR)
Preload

$(1 / 4+3 / 4$ DSC)
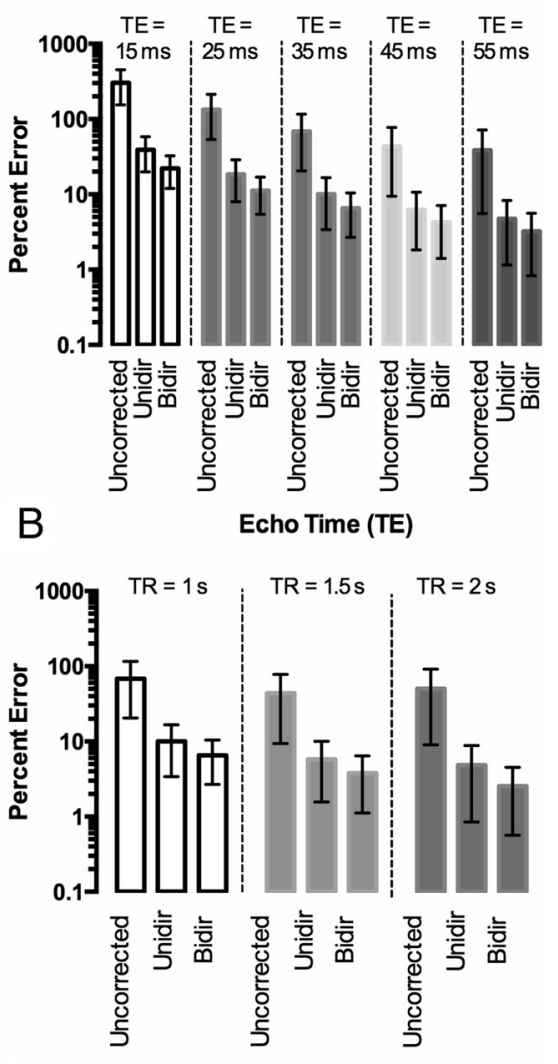

Repetition Time (TR)

FIG 2. Effect of TE and TR on CBV accuracy with flip angle $=60^{\circ}$. A, Percentage error in CBV estimation for different TEs by using TR $=1.0$ second without preload. $B$, Percentage error in CBV estimation for different TEs by using TR $=1.0$ second with $1 / 4$ dose preload. C, Percentage error in CBV estimation for different TRs with a $60^{\circ}$ flip angle and TE $=35 \mathrm{~ms}$ with no preload. $D$, Percentage error in CBV estimation for different TRs with a $60^{\circ}$ flip angle and TE $=35 \mathrm{~ms}$ with a $1 / 4$ dose preload.

$$
C_{\mathrm{p}}(t)=\frac{\rho}{k_{\mathrm{H}}} \times C B F \times \int_{0}^{t} C_{\alpha}(\tau) \times e^{-\frac{t-\tau}{M T T}} d \tau,
$$

where $\rho$ is the density of brain tissue $(1.04 \mathrm{~g} / \mathrm{mL})$, and $k_{\mathrm{H}}$ is the hematocrit difference between capillaries and large vessels $(0.73) .{ }^{17}$

The EES contrast agent concentration was computed by using a 2-compartment pharmacokinetic model as follows:

4) $C_{\mathrm{E}}(t)=K^{\mathrm{trans}} \times \int_{0}^{t} C_{\alpha}(\tau) \times e^{-\left(\frac{K^{\text {trans }}}{v_{e}}\right) \times(t-\tau)} d \tau$,

where $K^{\text {trans }}$ describes the efflux rate of contrast agent from the vasculature, often equated with permeability, and $K^{\text {trans }} / \nu_{\mathrm{e}}$ describes the rate of contrast agent influx back into the vasculature.

The relaxivity-time curves were obtained from Equation 1. For each relaxivity-time curve, the baseline signal was calculated as the median of the first 30 seconds of the signal.

\section{Tissue, Contrast Agent, and Noise Characteristics}

Specific tumor characteristics were estimated on the basis of previous data from Schmiedeskamp et al, ${ }^{17}$ including CBV $=5 \mathrm{~mL}$ /

$100 \mathrm{~g}, \mathrm{CBF}=60 \mathrm{~mL} / 100 \mathrm{~g} / \mathrm{min}$, and $T_{20}^{*}=0.05$ seconds. The blood volume fraction, $\mathrm{v}_{\mathrm{p}}$, was set equal to $\rho / k_{\mathrm{H}} \times$ $C B V$. Relaxivity values for gadobutrol (Gadavist; Bayer Schering Pharma, Berlin, Germany) were assumed to be $r_{1}=$ $3.6 \mathrm{mmol} / \mathrm{L}^{-1} \mathrm{~s}^{-1}{ }^{18}, r_{2, \mathrm{P}}{ }^{*}=87 \mathrm{mmol} /$ $\mathrm{L}^{-1} \mathrm{~s}^{-1},{ }^{19}$ and $r_{2, \mathrm{E}}^{*}=30 \mathrm{mmol} / \mathrm{L}^{-1} \mathrm{~s}^{-1}$. Monte Carlo simulations were performed by using the following values: $K^{\text {trans }}=0.214 \pm 0.04$ minute $^{-1}$ $($ range $=0.114-0.318), v_{\mathrm{e}}=0.722 \pm$ 0.17 (range $=0.259-0.985), T_{10}=$ $1.59 \pm 0.40$ seconds (range $=0.84-$ 2.87), $r_{2, \mathrm{P}}^{*}=87.0 \pm 17.4 \mathrm{mmol} / /^{-1} \mathrm{~s}^{-1}$ (range $=42.4-132)$, and $r_{2, \mathrm{E}}^{*}=30 \pm 6$ $\mathrm{mmol} / \mathrm{L}^{-1} \mathrm{~s}^{-1}($ range $=14.4-45.5) . \nu_{\mathrm{e}}$ was limited to a maximum of 1 , and $T_{10}$ was limited to a minimum of white matter (832 ms). ${ }^{20}$

$K^{\text {trans }}$ and $\nu_{\mathrm{e}}$ were chosen by using the average values and SDs from Zhang et al. ${ }^{21} T_{10}$ was estimated from variable flip angle mapping from 25 glioblastomas (5 precontrast T1 flip angle maps were acquired for each patient $\left[2^{\circ}, 5^{\circ}\right.$, $\left.10^{\circ}, 15^{\circ}, 30^{\circ}\right]$ ) and fitted by using a Levenberg-Marquardt nonlinear approach to the gradient-echo signal equation. The variances for $r_{2, \mathrm{P}}^{*}$ and $r_{2, \mathrm{E}}^{*}$ are, to the best of our knowledge, not well-defined in the literature and were chosen to be $20 \%$ to approximately match the SDs of the other parameters. $K_{\mathrm{p}}$, the susceptibility calibration factor, was chosen to generate a $40 \%$ peak signal drop in gray matter, for which $\mathrm{CBF}=60 \mathrm{~mL} / 100 \mathrm{~g} / \mathrm{min}$ and $\mathrm{CBV}=4 \mathrm{~mL} / 100$ g were chosen. ${ }^{22}$ The whole-brain average was selected as the average of 1000 white matter voxels (including noise), with $\mathrm{CBF}=$ $25 \mathrm{~mL} / 100 \mathrm{~g} / \mathrm{min}$ and $\mathrm{CBV}=2 \mathrm{~mL} / 100 \mathrm{~g}$.

Contrast-to-noise ratio (CNR) was first measured in a sample of 25 human glioblastomas (flip angle $=35^{\circ}, \mathrm{TE}=32 \mathrm{~ms}$, and $\mathrm{TR}=1.8$ seconds), which had a CNR of 40.5. To model noise added by the TE and TR used, we scaled the CNR by $C \times \sin (\alpha) \times e^{-T E / T_{2}^{*}}\left(1-e^{-T R / T_{1}}\right) / \sin \left(35^{\circ}\right) \times e^{-0.032} /$ $T_{2}^{\star}\left(1-e^{-1.8 / T_{1}}\right)$, where the numerator incorporates the dose (C) of the new protocol and TE, TR, the flip angle, and the denominator scales the CNR according to the parameters used in acquiring the human data.

The CNR, which is defined with the following equation, was used to calculate $\mathrm{SD}^{23,24}$ :

$$
C N R=\frac{\Delta R_{2, \max }^{\star}}{\sigma}
$$

where $\Delta R_{2, \max }^{*}$ is the maximum value of $\Delta R_{2}^{*}$ and $\sigma$ is the SD of the Gaussian noise added to each time point. Gaussian noise was added with mean of zero and a SD of $\sigma$. 


\section{Leakage Correction Algorithms}

Uncorrected CBV was computed by integrating $\Delta R_{2}^{*}(t)$, while leakage-corrected CBV was obtained by using either Unidir ${ }^{10}$ or Bidir $^{11,13}$ leakage-correction algorithms. The "ground truth" $\left(\Delta R_{2}^{*}(t)_{\mathrm{gt}}\right)$ estimate of CBV was calculated under conditions of no noise with $K^{\text {trans }}=0$. The percentage error from ground truth was calculated for uncorrected and leakage-corrected CBV estimates with added noise.

\section{Effects of Preload Incubation Time and Truncation of the DSC Time-Series}

To estimate the effects of preload incubation time, we compared estimates of CBV with delays of 5-10 minutes between preload and bolus injection. To estimate the effects of truncating $\Delta R_{2}^{\star}(t)$ on CBV estimates, we compared CBV estimates by using the first $30,60,90$, or 120 seconds of the postbaseline $\Delta R_{2}^{*}(t)$ as well as the entire 150-second data.

\section{Monte Carlo Simulations to Estimate CBV Confidence Intervals}

For each set of pulse sequence parameters, Gaussian noise was added to each time point with normal distribution (zero mean, $\mathrm{SD}$ equal to the maximum signal scaled by the CNR), and tumor characteristics were generated according to the normal distributions described previously. A Monte Carlo simulation was conducted by using 250 randomly chosen tumors, with random noise, for each set of pulse sequence parameters. Percentage error was calculated by using the computed CBV and the ground truth CBV. The 95\% confidence intervals of percentage error were subsequently generated for the uncorrected CBV, and each of the leakage-correction algorithms and are shown in each of the figures. For Figs 1-3, 1 particular protocol (flip angle $=60^{\circ}, \mathrm{TE}=35$ $\mathrm{ms}, \mathrm{TR}=1.0 \mathrm{~s}, 1 / 4$ preload dose $+3 / 4$ DSC-MR imaging, waiting time $=5$ minutes) was chosen as the template based on American Society of Functional Neuroradiology recommendations, ${ }^{25}$ with variations to only 1 of the parameters shown for each subfigure. For Fig 4, all combinations of flip angle, TR, TE, and preload dosage were evaluated. For all figures, integration of the relaxivity-time curve was performed from the injection time point to the end (2.5 minutes), unless otherwise noted.

\section{RESULTS}

Without preload, there is reduced T1-weighting and increased T2*-weighting with smaller flip angles as manifested by higher $\Delta R_{2}^{*}(t)$, best seen in the "tail" (On-line Fig $1 A$ ). Preload administration increases $2^{\star}$-weighting of the signal (On-line Fig $1 B$ ). In this case, without preload, the $35^{\circ}$ relaxivity-time curve is closest to ground truth $\left(\Delta R_{2}^{\star}(t)_{\mathrm{gt}}\right)$, while the $60^{\circ}$ and $35^{\circ}$ curves are equally close to the truth curve after preload ( $1 / 4$ dose $+3 / 4$ dose DSC-MR imaging). On the basis of the formula used for CNR, the $35^{\circ}$ flip angle also yielded the most noise, as exemplified in the preload DSC-MR imaging curve. For both nonpreload and preload administration, Unidir-corrected $\Delta R_{2}^{*}(t)$ varied more from $\Delta R_{2}^{\star}(t)_{\mathrm{gt}}$ across all tested flip angles compared with Bidir-corrected $\Delta R_{2}^{*}(t)$, particularly right after the first pass of the bolus (On-line Fig $1 C-F$ ). Figure $1 A,-B$ illustrates the percentage errors for uncorrected, Unidir, and Bidir CBV estimates, compared with
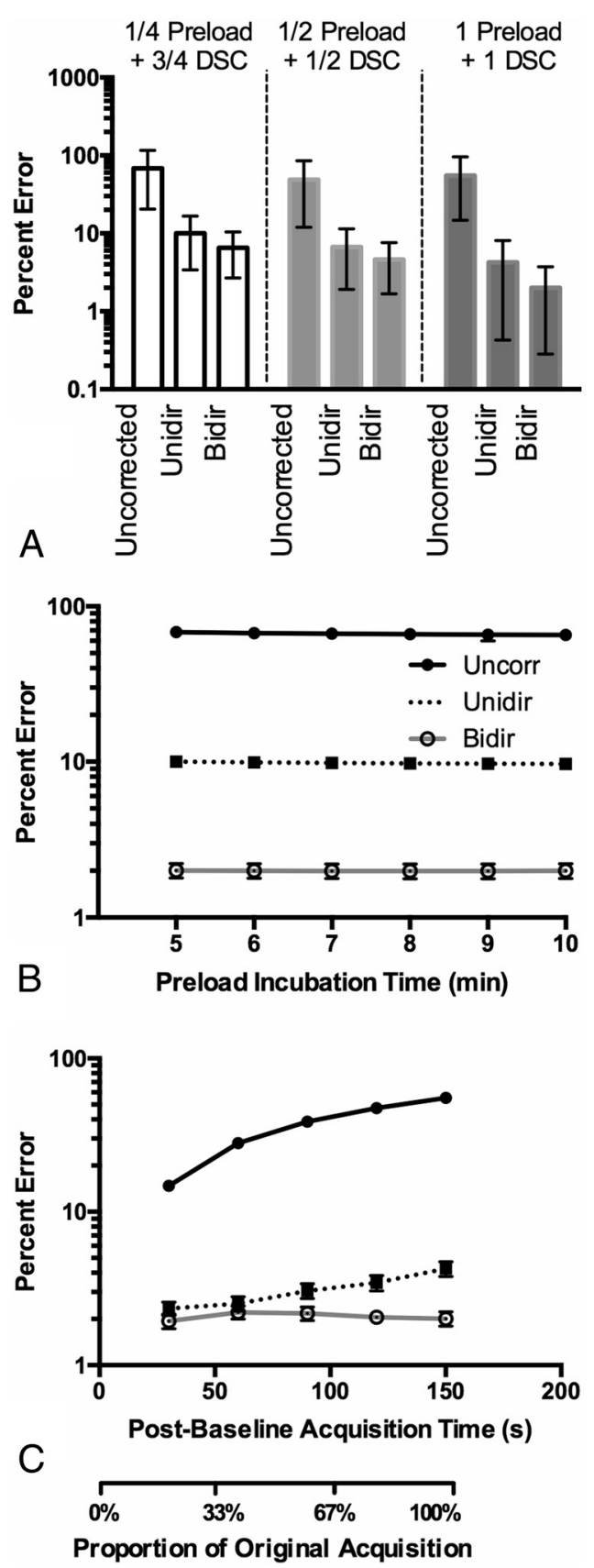

FIG 3. Effects of preload dosage, incubation time, and truncation of corrected $\Delta R_{2}^{\star}(t)$ on CBV fidelity. $A$, Percentage errors in CBV for each preload dosage with $95 \% \mathrm{Cl}$. The best performance was obtained with 1-dose preload and 1-dose DSC-MR imaging bolus when using TE $=35$ $\mathrm{ms}, \mathrm{TR}=1.0$ second, and flip angle $=60^{\circ}$. Note the increased T2*weighting from a full-dose preload decreases the CBV error when using these acquisition parameters. $B$, Effects of preload incubation time on CBV estimation when using preload. C, Effects of truncation of the $\Delta R_{2}^{\star}(t)$ curves and leakage-correction strategies on CBV estimation when using a 1-dose preload followed by a 1-dose DSC bolus injection.

$\Delta R_{2}^{\star}(t)_{\mathrm{gt}}$, for different flip angles. With this particular combination of TR/TE/preload dosage, the $35^{\circ}$ flip angle had the lowest error. Furthermore, error after both Unidir and Bidir leakage corrections tracked with error in the uncorrected CBV (ie, the lower error in uncorrected CBV corresponded with lower error after leakage correction). For all tested flip angles, uncorrected CBV 

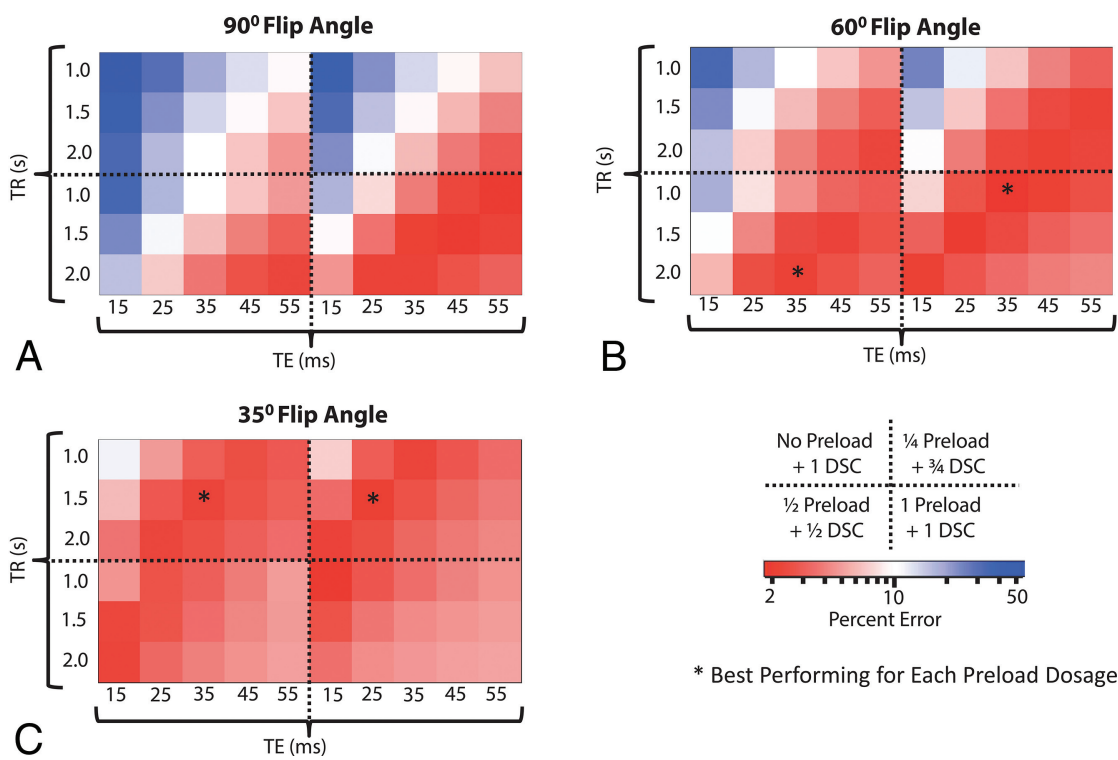

* Best Performing for Each Preload Dosage

FIG 4. Heat map diagrams depicting the percentage error in the CBV estimation for combinations of acquisition protocols with Bidir. Each quadrant within each subfigure represents a different preload dose. Each subfigure represents a different flip angle: $90^{\circ}(A), 60^{\circ}(B)$, and $35^{\circ}(C)$. Optimal strategies balance T1-weighted and $\mathrm{T} 2^{*}$-weighted effects from contrast agent extravasation via a combination of TE, TR, and preload. Red boxes indicate acquisition protocols with minimum error, while blue boxes indicate protocols with poor CBV fidelity (high error compared with ground truth).

estimates had the highest error, followed by the Unidir and then the Bidir estimates.

Results indicate longer TEs increase the $\mathrm{T} 2{ }^{\star}$-weighting of $\Delta R_{2}^{\star}(t)$ (On-line Fig $2 A,-B$; flip angle $=60^{\circ}, \mathrm{TR}=1.0$ second). Without preload (Fig $2 A$ ), $\mathrm{TE}=55 \mathrm{~ms}$ yielded the most accurate $\Delta R_{2}^{\star}(t)$ for all 3 correction strategies by using all leakage-correction strategies. With $1 / 4$ dose preload (Fig $2 B$ ), TE $=45-55 \mathrm{~ms} \mathrm{had}$ smaller error than without preload, though the $55 \mathrm{~ms}$ performed slightly better. Post hoc leakage-correction error tracked with uncorrected error in these examples. Results also suggest an increased T2*-weighting (or decreased T1-weighting) with longer TRs (On-line Fig $2 C,-D$ ). Independent of preload, TR $\geq 1.5 \mathrm{sec}-$ onds yielded $\Delta R_{2}^{\star}(t)$ with less error compared with $\Delta R_{2}^{*}(t)_{\mathrm{gt}}$ for a $60^{\circ}$ flip (Fig $2 C,-D$ ) for the chosen flip angle, TE, and preload dosage. In general, CBV error with the 3 methods was linearly correlated.

Preload primarily increases $\mathrm{T} 2{ }^{*}$-weighting and reduces $\mathrm{T} 1$ weighting in $\Delta R_{2}^{*}(t)$ (On-line Fig $3 A$; flip angle $=60^{\circ}, \mathrm{TE}=35 \mathrm{~ms}$, $\mathrm{TR}=1.5$ seconds). For these parameters, 1 preload +1 bolus dosing yielded higher $\Delta R_{2}^{*}(t)$ fidelity compared with ground truth $\Delta R_{2}^{*}(t)$ than the $1 / 4+3 / 4$ and $1 / 2+1 / 2$ dosing schemes (Fig $3 A$ ). Even though the $1 / 2+1 / 2$ and $1+1$ dosing schemes had approximately the same uncorrected CBV percentage error, the post hoc leakagecorrection algorithms benefited from the higher CNR that the full DSC-MR imaging dose provides. Results also suggest that preload does not act by decreasing the concentration-dependent rate of contrast agent efflux, but rather by decreasing baseline tissue T1 before bolus injection, as well as increasing $\mathrm{T} 2{ }^{*}$-weighting, as evidenced by identical wash-in rates and concentration-dependent reductions in baseline T1 (On-line Fig 3B, $-C$ ).

With incubation times of 5-10 minutes, the change in CBV error is virtually similar, with a slight, gradual decrease in error from 5 to 10 minutes (Fig 3B). Next, because CBV is computed from the integration of $\Delta R_{2}^{\star}(t), 1$ strategy for mitigating leakage effects is truncating $\Delta R_{2}^{\star}(t)$ after the first pass. As expected, the less data used for computing CBV, the lower is the percentage error for uncorrected CBV. For Unidir CBV, mean percentage error is lowest when 30 seconds is used and gradually increases with time. For Bidir CBV, percentage error was approximately the same for all cutoff times (Fig 3C).

The protocol with the lowest overall mean percentage error used a $60^{\circ} \mathrm{flip}$ angle, TE/TR $=35 / 1000 \mathrm{~ms}$ with 1 dose preload, by using the bidirectional correction; however, there were multiple protocols whose 95\% CIs overlapped (On-line Table), suggesting there are several strategies that could be used to get similar CBV estimates. In general, the best performing strategies (dark red areas in Fig 4 and On-line Fig 4) were those that balanced both $\mathrm{T} 1-$ and $\mathrm{T} 2{ }^{*}$ weighting secondary to contrast agent extravasation, with a mean uncorrected CBV error of $<70 \%$ for all the "optimal" strategies with 1 total dose of contrast and $<80 \%$ for those with 2 total doses of contrast agent, as opposed to a much larger error for other protocols. Preload did not necessarily depress percentage error, as evidenced by the $35^{\circ}$ flip angle, in which higher preload dosages could "overshoot" the "ground truth." The acquisition strategies with the lowest mean error in this simulation (flip angle/TE/TR) for each preload dosing were the following: 1) with no preload and full dose for DSC-MR imaging: $35^{\circ} / 35 \mathrm{~ms} / 1.5$ seconds; 2 ) with $1 / 4$ dose preload and $3 / 4$ dose bolus: $35^{\circ} / 25 \mathrm{~ms} / 1.5$ seconds; 3 ) with $1 / 2$ dose preload and $1 / 2$ dose bolus: $60^{\circ} / 35 \mathrm{~ms} / 2.0$ seconds; and 4) with 1-dose preload and 1-dose bolus: $60^{\circ} / 35 \mathrm{~ms} / 1.0$ second. The $90^{\circ}$ flip angle only appeared as an optimal strategy with 1-dose preload and 1-dose bolus.

\section{DISCUSSION}

The purpose of this study was to evaluate the influence of various DSC-MR imaging acquisition strategies and post hoc leakagecorrection algorithms on the fidelity of the estimation. In general, the performance of both leakage-correction algorithms improves as the leakage-contaminated $\Delta R_{2}^{*}(t)$ curve more closely approximates $\Delta R_{2}^{*}(t)_{\mathrm{gt}}$. Furthermore, a much more "homogeneous" performance is seen for the double dose because many more protocols were "optimal" with the double dose. This finding would seem to imply that this scheme is less sensitive to the physiologic variations that would impact the estimates of $\Delta R_{2}^{*}(t)$.

As has been established previously, increased $\mathrm{T} 2{ }^{\star}$-weighted leakage results from a lower flip angle, longer TR, longer TE, and higher preload dosage. ${ }^{15,26}$ Given that the errors before and after leakage correction are generally correlated, the optimal strategies minimize errors in uncorrected rCBV, which can be accom- 
plished by balancing T1- and T2*-weighted leakage effects. Therefore, the optimal protocols balance these 2 opposing effects so that the DSC-MR imaging curves do not deviate too much from the ground truth. For example, a TR of 1.0 second, which is relatively T1-weighted, can be offset with a full dose of preload, which is quite $\mathrm{T} 2{ }^{\star}$-weighted. Some of the optimal protocols, on the other hand, take a middle-of-the-road approach (ie, $60^{\circ}$ flip angle or $\mathrm{TE}=35 \mathrm{~ms}$ ) so that none of the parameters cause the DSC-MR imaging curves to be overly affected by T1-weighted or T2*weighted leakage effects.

Our results also suggest that the mechanism by which preload increases CBV fidelity is by increasing $\mathrm{T} 2{ }^{\star}$-weighting and decreasing T1-weighting of the $\Delta R_{2}^{\star}(t)$ curves rather than by decreasing the flux of contrast agent diffusion into the EES. While preload and postprocessing leakage-correction algorithms have been shown to work synergistically in many cases, including intermediate-to-high flip angle acquisitions, ${ }^{9}$ it is also possible for preload to overcompensate and worsen the deviation of the leakage-contaminated $\Delta R_{2}^{*}(t)$ curve compared with the ground truth; Hu et $\mathrm{al}^{27}$ drew the same conclusion. Furthermore, sufficient preload correction was found between 6 and 10 minutes, which agrees with the findings of $\mathrm{Hu}$ et $\mathrm{al}^{27}$ and Kassner et $\mathrm{al}^{28}$

The bidirectional leakage correction accounts for backward flux of contrast agent and was shown to reduce CBV error compared with the unidirectional leakage correction in all 180 acquisition scenarios tested. Both leakage-correction algorithms work by first performing a least-squares fit of the model-generated corrected $\Delta R_{2}^{\star}(t)$ curve plus the leakage term, and then subtracting the original $\Delta R_{2}^{\star}(t)$ curve by the calculated leakage term. Thus, if the computed leakage term does not include back flux of contrast agent, it can cause the corrected $\Delta R_{2}^{\star}(t)$ curve to adopt a shape noticeably different from $C_{\mathrm{p}}$ (Equation 3 ), thereby overestimating and underestimating the ground truth curve immediately following the first pass of the bolus. Thus, CBV estimates obtained by using the unidirectional algorithm have approximately twice the error compared with estimates obtained by using the bidirectional leakage-correction algorithm.

The study has several notable limitations. First, we did not account for errors arising from either variations in MTT (including bolus dispersion) or bolus delay, both of which would serve to increase the percentage error for the leakage-correction algorithms presented in the study due to MTT sensitivity. Therefore, the percentage errors are meant to be relative, with many of the optimal protocols providing a balance between $\mathrm{T} 1$ and $\mathrm{T} 2{ }^{\star}$ leakage effects over the population of tumors. Another limitation is the lack of more sophisticated integration of all of the effects of microvascular and microcellular morphologies on DSC-MR imaging data, including, but not limited to, the arbitrary geometry of the underlying vessels. ${ }^{29,30}$ Furthermore, the noise modeled does not take into account potential sources, such as coil quality, section thickness, and the use of a global AIF. Much of the population data used for these tumors were acquired in small cohorts, and a study in a larger population would be required for more accurate modeling of the tumor population characteristics, particularly $r_{2}^{*}$. Finally, the results in the simulation warrant validation in real patients. Testing every combination of MR imaging protocol is infeasible in real patients. Furthermore, it is currently impossible to ascertain the ground truth $\mathrm{rCBV}$; however, these results could be validated in real brain tumors by using an extension of the Paulson and Schmainda ${ }^{4}$ methodology, in which the top performing protocols are tested against each other for variability between scans.

\section{CONCLUSIONS}

The current study demonstrates that the choice of image acquisition and preload dosing and/or fractionation has tremendous impact on the fidelity of CBV estimation. Results suggest that a variety of acquisition strategies can be used to obtain similar accuracy of CBV estimation, while the bidirectional leakage-correction algorithm aids in minimizing errors in CBV estimation under all scenarios. To compute the most accurate CBV, one should focus on standardizing a DSC-MR imaging acquisition strategy that minimizes errors in the underlying leakage-contaminated $\Delta R_{2}^{*}(t)$ curve by balancing $\mathrm{T} 1$ and $\mathrm{T} 2{ }^{*}$ contamination effects over the tumor population, which will, in turn, reduce the residual errors in CBV estimation following leakage correction.

Disclosures: Benjamin M. Ellingson—UNRELATED: Consultancy: MedQIA, Siemens, Roche, Agios Pharmaceuticals, Bristol-Meyer Squib, Nativis, Insys Therapeutics, Omniox; Grants/Grants Pending: Siemens, Roche.

\section{REFERENCES}

1. Meier P, Zierler KL. On the theory of the indicator-dilution method for measurement of blood flow and volume. J Appl Physiol 1954;6: 731-44 Medline

2. Essig M, Nguyen TB, Shiroishi MS, et al. Perfusion MRI: the five most frequently asked clinical questions. AJR Am J Roentgenol 2013; 201:W495-510 CrossRef Medline

3. Petrella JR, Provenzale JM. MR perfusion imaging of the brain: techniques and applications. AJR Am J Roentgenol 2000;175:207-19 CrossRef Medline

4. Paulson ES, Schmainda KM. Comparison of dynamic susceptibilityweighted contrast-enhanced MR methods: recommendations for measuring relative cerebral blood volume in brain tumors. Radiology 2008;249:601-13 CrossRef Medline

5. Cha S, Knopp EA, Johnson G, et al. Intracranial mass lesions: dynamic contrast-enhanced susceptibility-weighted echo-planar perfusion MR imaging. Radiology 2002;223:11-29 CrossRef Medline

6. Vonken EJ, van Osch MJ, Bakker CJ, et al. Measurement of cerebral perfusion with dual-echo multi-slice quantitative dynamic susceptibility contrast MRI. J Magn Reson Imaging 1999;10:109-17 Medline

7. Schmiedeskamp H, Straka M, Newbould RD, et al. Combined spinand gradient-echo perfusion-weighted imaging. Magn Reson Med 2012;68:30-40 CrossRef Medline

8. Skinner JT, Robison RK, Elder CP, et al. Evaluation of a multiple spin- and gradient-echo (SAGE) EPI acquisition with SENSE acceleration: applications for perfusion imaging in and outside the brain. Magn Reson Imaging 2014;32:1171-80 CrossRef Medline

9. Boxerman JL, Prah DE, Paulson ES, et al. The role of preload and leakage correction in gadolinium-based cerebral blood volume estimation determined by comparison with MION as a criterion standard. AJNR Am J Neuroradiol 2012;33:1081-87 CrossRef Medline

10. Boxerman JL, Schmainda KM, Weisskoff RM. Relative cerebral blood volume maps corrected for contrast agent extravasation significantly correlate with glioma tumor grade, whereas uncorrected maps do not. AJNR Am J Neuroradiol 2006;27:859-67 Medline

11. Leu K, Boxerman JB, Cloughesy TF, et al. Improved leakage correction for single-echo dynamic susceptibility contrast (DSC) perfusion MRI estimates of relative cerebral blood volume (rCBV) in 
high-grade gliomas by accounting for bidirectional contrast agent exchange. AJNR Am J Neuroradiol 2016;37:1440-46

12. Bjornerud A, Sorensen AG, Mouridsen K, et al. T1- and T2*-dominant extravasation correction in DSC-MRI, Part I, theoretical considerations and implications for assessment of tumor hemodynamic properties. J Cereb Blood Flow Metab 2011;31:2041-53 CrossRef Medline

13. Leu K, Boxerman JL, Lai A, et al. Bidirectional contrast agent leakage correction of dynamic susceptibility contrast (DSC)-MRI improves cerebral blood volume estimation and survival prediction in recurrent glioblastoma treated with bevacizumab. J Magn Reson Imaging 2016;44:1229-37 CrossRef Medline

14. Ostergaard L, Weisskoff RM, Chesler DA, et al. High resolution measurement of cerebral blood flow using intravascular tracer bolus passages, Part I: mathematical approach and statistical analysis. Magn Reson Med 1996;36:715-25 CrossRef Medline

15. Quarles CC, Gochberg DF, Gore JC, et al. A theoretical framework to model DSC-MRI data acquired in the presence of contrast agent extravasation. Phys Med Biol 2009;54:5749-66 CrossRef Medline

16. Simpson NE, He Z, Evelhoch JL. Deuterium NMR tissue perfusion measurements using the tracer uptake approach, I: optimization of methods. Magn Reson Med 1999;42:42-52 Medline

17. Schmiedeskamp H, Andre JB, Straka M, et al. Simultaneous perfusion and permeability measurements using combined spin- and gradient-echo MRI. J Cereb Blood Flow Metab 2013;33:732-43 CrossRef Medline

18. Pintaske J, Martirosian $\mathrm{P}$, Graf $\mathrm{H}$, et al. Relaxivity of gadopentetate dimeglumine (Magnevist), gadobutrol (Gadovist), and gadobenate dimeglumine (MultiHance) in human blood plasma at 0.2, 1.5, and 3 Tesla. Invest Radiol 2006;41:213-21 CrossRef Medline

19. Kjolby BF, Østergaard L, Kiselev VG. Theoretical model of intravascular paramagnetic tracers effect on tissue relaxation. Magn Reson Med 2006;56:187-97 CrossRef Medline

20. Wansapura JP, Holland SK, Dunn RS, et al. NMR relaxation times in the human brain at 3.0 Tesla. J Magn Reson Imaging 1999;9:531-38 Medline

21. Zhang N, Zhang L, Qiu B, et al. Correlation of volume transfer coefficient Ktrans with histopathologic grades of gliomas. J Magn Reson Imaging 2012;36:355-63 CrossRef Medline
22. Wu O, Østergaard L, Koroshetz WJ, et al. Effects of tracer arrival time on flow estimates in MR perfusion-weighted imaging. Magn Reson Med 2003;50:856-64 CrossRef Medline

23. van Osch MJ, Vonken EJ, Viergever MA, et al. Measuring the arterial input function with gradient echo sequences. Magn Reson Med 2003;49:1067-76 CrossRef Medline

24. Vonken EP, van Osch MJ, Bakker CJ, et al. Simultaneous quantitative cerebral perfusion and Gd-DTPA extravasation measurement with dual-echo dynamic susceptibility contrast MRI. Magn Reson Med 2000;43:820-27 Medline

25. Welker K, Boxerman J, Kalnin A, et al; American Society of Functional Neuroradiology MR Perfusion Standards and Practice Subcommittee of the ASFNR Clinical Practice Committee. ASFNR recommendations for clinical performance of MR dynamic susceptibility contrast perfusion imaging of the brain. AJNR Am J Neuroradiol 2015;36:E41-51 CrossRef Medline

26. Liu HL, Wu YY, Yang WS, et al. Is Weisskoff model valid for the correction of contrast agent extravasation with combined $\mathrm{T} 1$ and T2 ${ }^{\star}$ effects in dynamic susceptibility contrast MRI? Med Phys 2011; 38:802-09 CrossRef Medline

27. Hu LS, Baxter LC, Pinnaduwage DS, et al. Optimized preload leakage-correction methods to improve the diagnostic accuracy of dynamic susceptibility-weighted contrast-enhanced perfusion MR imaging in posttreatment gliomas. AJNR Am J Neuroradiol 2010;31: 40-48 CrossRef Medline

28. Kassner A, Annesley DJ, Zhu XP, et al. Abnormalities of the contrast re-circulation phase in cerebral tumors demonstrated using dynamic susceptibility contrast-enhanced imaging: a possible marker of vascular tortuosity. J Magn Reson Imaging 2000;11:103-13 Medline

29. Pathak AP, Ward BD, Schmainda KM. A novel technique for modeling susceptibility-based contrast mechanisms for arbitrary microvascular geometries: the finite perturber method. Neuroimage 2008;40:1130-43 CrossRef Medline

30. Semmineh NB, Xu J, Boxerman JL, et al. An efficient computational approach to characterize DSC-MRI signals arising from three-dimensional heterogeneous tissue structures. PLoS One 2014;9: e84764 CrossRef Medline 